(REVIEW ARTICLE)

\title{
Treating developmental dyslexia using transcranial stimulation combined with a reading stimulation program: A pilot study
}

\author{
Isabela Ciola de Castro ${ }^{1,}{ }^{*}$, Mauro Muszkat ${ }^{2}$, Vânia Carvalho Lima ${ }^{1}$, Sueli Rizzutti ${ }^{2}$ and Clara Brandão de Ávila ${ }^{1}$ \\ ${ }^{1}$ Department of speech-language and hearing sciences - Paulista School of Medicine- Federal University of São Paulo, \\ Address: Rua Botucatu, 802 - Vila Clementino- 04023-062 - São Paulo - Brazil. \\ 2 Department of Psychobiology - Federal University of São Paulo, R. Napoleão de Barros, 925, 04021002 - São Paulo - \\ Brazil.
}

World Journal of Biology Pharmacy and Health Sciences, 2021, 07(03), 080-088

Publication history: Received on 19 August 2021; revised on 25 September 2021; accepted on 27 September 2021

Article DOI: https://doi.org/10.30574/wjbphs.2021.7.3.0105

\begin{abstract}
Objective: Evaluate the effect of transcranial direct current stimulation (tDCS) combined with reading therapy on the performance of reading tasks in a serie of cases of children and adolescents with developmental dyslexia (DD).

Method: (IRB/UNIFESP number:1281/2017). Four dyslexic students (two children and two adolescents) were evaluated with regards to rate and accuracy of reading fluency on three occasions: pre-intervention as well as one and six months after intervention. The intervention consisted of five consecutive sessions of active tDCS (1 mA for 20 minutes), followed by a two-day interval and another five sessions of tDCS combined with cognitive skills stimulation of phonological processing and decoding of words. Anodal tDCS was administered over the left parieto-occipital cortex, with the cathode placed over contralateral deltoid muscle. Each session was preceded by execution of semantic-lexical and phonological evocation tasks. Data were analyzed using Friedman test and JT method for analysis of clinical significance and the reliable change index.
\end{abstract}

Results: Improvements were found in text reading rate and accuracy and word accuracy at the one-month and sixmonth evaluation. A significant increase was found in correct reading of words and pseudowords six months after intervention. Most of the improvements were reliable, with clinical significance in some cases. Conclusion: Transcranial direct current stimulation combined with a reading stimulation program proved effective at improving reading skills in these participants.

Keywords: Dyslexia; Transcranial Direct Current Stimulation; Reading; Longitudinal Study

\section{Introduction}

Developmental dyslexia (DD) are a neurobiological disorder characterized mainly by an impaired ability to decode and automatically, precisely recognize written words [1]. Magnetic resonance studies confirm abnormal and/or hypoactivation of temporo-occipital, temporo-parietal, left lower parietal, as well as the anterior and posterior cingulate regions (posterior areas of the left brain [2,3]. One study also found greater activation of the temporal region of right hemisphere in individuals with DD [3].

Current studies describe different types of dyslexia [4,5]. Moreover, due to the complexity of cognitive-linguistic processes involved in reading, several clinical manifestations are reported, such as lower performance on lexical

\footnotetext{
${ }^{*}$ Corresponding author: Isabela Ciola de Castro

Department of speech-language and hearing sciences - Paulista School of Medicine - Federal University of São Paulo, Address: Rua Botucatu, 802 - Vila Clementino- 04023-062 - São Paulo - Brazil.
}

Copyright (C) 2021 Author(s) retain the copyright of this article. This article is published under the terms of the Creative Commons Attribution Liscense 4.0. 
decision tasks or fast, precise reading of words during activities of morphosyntactic awareness and orthographic processing [6-8]. Such problems in the performance of low cognitive order tasks are generally related to deficits in phonological processing, which encompasses skills such as short-term and operational phonological memory, automatic phonological access to lexicon, and phonological awareness [9].

The complexity of processes and brain circuits involved in reading suggests that the rehabilitation of these decoding abnormalities requires therapeutic methods with multisensory and multilevel approach. In line with such approaches, programs have been designed to promote stimulation of different skills involved in learning to read, such as visual skills for recognition of letters, cognitive and language skills for processing phonological information and the phonemegrapheme association, automatic recognition of written words, and text comprehension [10,11]. Motor stimulation and balance tasks also contribute to the development of spatiotemporal orientation, rhythm, and motor coordination, which are essential to reading and writing, considering the role of the cerebellum in the optimization and automatization of language [12].

Recently, researchers have studied the use of novel resources for rehabilitation of neurodevelopmental disorders, such as transcranial direct current stimulation (tDCS) [13-17]. This therapeutic modality involves noninvasive low-intensity electrical stimulation over the scalp with the aim of modulating cortical excitability by changing the membrane potential of cells located in the cortex and either exciting (anodal stimulation) or inhibiting (cathodal stimulation) desired areas $[14,17]$.

Researchers have studied effects and durability after applying tDCS combined with interventions involving stimulation of specific functions and skills in different neurodevelopmental disorders, including reading [14-16] in children and adolescents [14-17].

With regards to reading, some studies have found improvements in recognition of less common words and non-words in children and adolescents with DD after a short intervention involving anodal stimulation of posterior areas of left brain. Such effects were found in both short and long terms [14-16].

However, there is insufficient scientific evidence regarding effects of tDCS on DD and its combination with stimulation therapies of another nature (such as, linguistic) in children and adolescents with DD. Additionally, although most studies have applied the anodal electrode over temporo-parietal region, there is no consensus concerning the best placement of electrodes, as the different anodal and cathodal montages employed have achieved different results [14-16]. There is also no agreement about the need for and what therapeutic reading stimulation therapies should be used during tDCS $[14,15,33]$.

The hypothesis tested in the present study was that anodal tDCS administered over left parieto-occipital region, which is related to the visual areas of reading, combined with multisensory, multilevel therapy involving the stimulation of cognitive and reading skills, would produce positive effects on rate and accuracy of reading fluency in students with DD.

Therefore, the aim of the present pilot study was to investigate the effects of anodal tDCS administered over left parietooccipital region combined with a multilevel reading stimulation program on rate and accuracy of reading fluency in a case series of children and adolescents with DD.

\section{Methods}

The present prospective, longitudinal study with quantitative and qualitative analysis received approval from the local institutional review board (CEP-UNIFESP/EPM - certificate number: 1281/2017).

Four students with a multidisciplinary diagnosis of DD [two children (both girls with a mean age of 8,9) and two adolescents (one girl and one boy with a mean age of 13,4) participated in the study. The students attended the $3^{\text {rd }}$ (private system), $4^{\text {th }}, 7^{\text {th }}$, and $8^{\text {th }}$ (public system) grades of primary school. All participants were submitted to phonological and neuropsychological evaluations to ensure that they met the inclusion criteria: adequate oral comprehension; reading speed and accuracy of texts and isolated words below the average and standard deviation; average or above average intelligence quotient; absence of neuropsychiatric comorbidities; signed statement of informed consent by a legal guardian and statement of consent signed by the child or adolescent.

The flowchart of the study is displayed in Figure 1. 


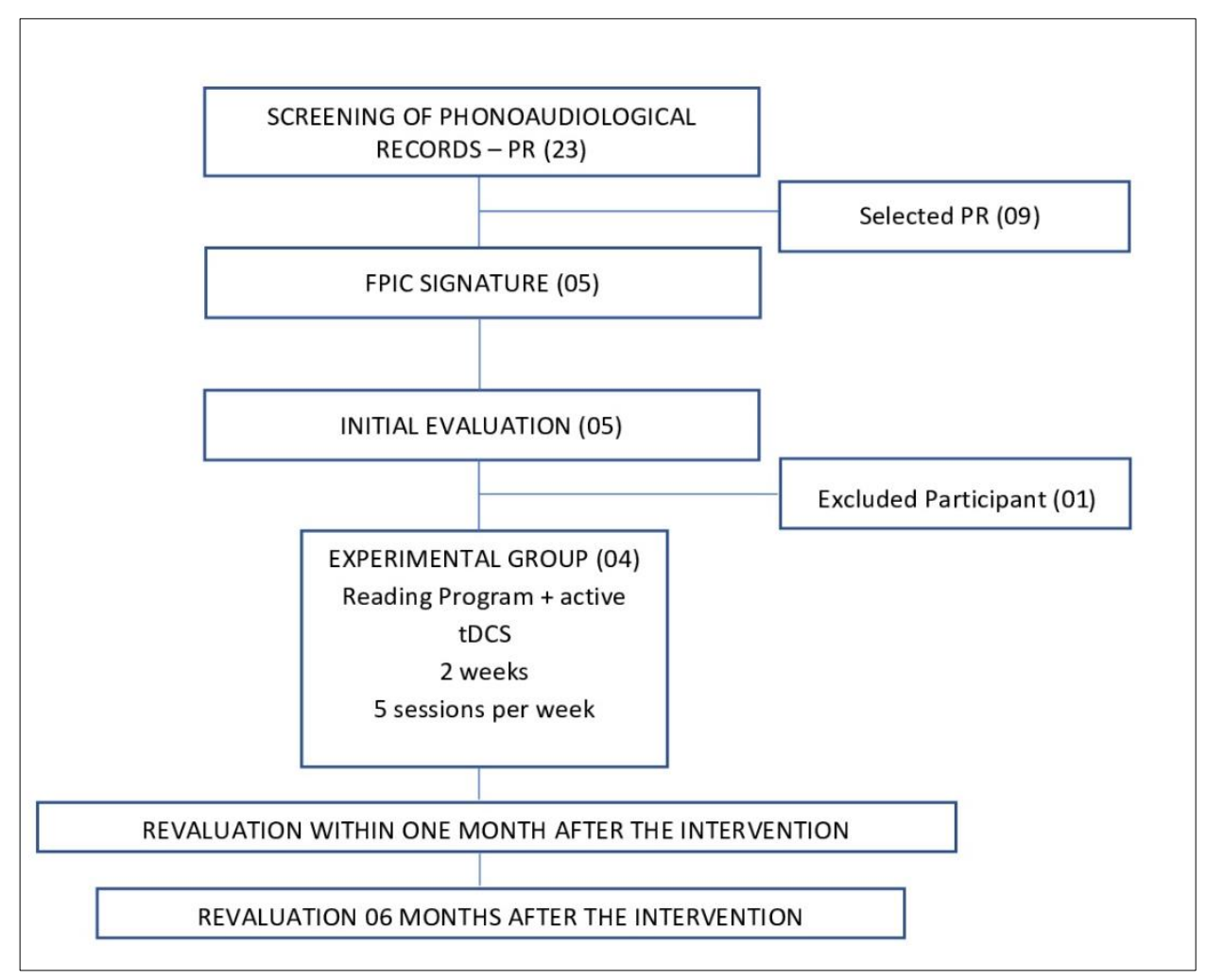

Figure 1 Study flow chart

\subsection{Evaluation protocol}

Speech therapists unaware of the study monitored the evolution of the participants, assessing reading skills on three occasions: pre-intervention, one month after stimulation, and six months after simulation.

The following were the variables of interest:

Fluency reading words [18] and narrative text [19]: number of words read per minute (rate) and read correctly (accuracy).

- Accuracy/score [20]: number of words read correctly per psycholinguistic category (20 common words, 20 uncommon words, and 20 pseudowords) and total.

\subsection{Intervention protocol}

\subsubsection{Transcranial direct current stimulation}

A tDCS device (Soterix Medical Inc.) was used with two sponge (non-metallic) surface electrodes $\left(5 \times 7 \mathrm{~cm}^{2}\right)$ moistened with saline solution. A current of $1 \mathrm{~mA}\left(0.029 \mathrm{~mA} / \mathrm{cm}^{2}\right)$ was administered for 20 minutes with the anodal electrode positioned over the left parieto-occipital cortex (10/20 international electroencephalogram system) and the cathode over contralateral deltoid muscle.

\subsubsection{Multilevel reading stimulation program}

All participants were submitted to tDCS combined with a reading stimulation program, which was designed based on a bibliographic survey of the most common difficulties found in children with DD and skills stimulated in different DD intervention programs.

- Prior to the placement of the electrodes for tDCS, stimulation of semantic and phonological verbal fluency was performed with the participant on a balance disk (mean duration: 3 minutes). The aim of this activity was the stimulation of executive functions and language functions (lexical access and vocabulary) and the activation of balance pathways $[21,22]$. After stepping onto the balance disk, the participant was instructed to list the 
maximum number of words in a particular semantic class for one minute and the maximum number of words beginning with a given phoneme for one minute. Assistance was given in the form of semantic and/or phonological cues.

- During tDCS (duration: 20 minutes), stimulation of phonological processing (phonological awareness, operational memory, and rapid naming) along with phonological and lexical routes for reading, as described below:

* Stimulation of phonological awareness: syllabic and phonemic exclusion skills [23]. After hearing a word, the participant was instructed to exclude a syllable or phoneme and say the resulting word. An example was given and, when necessary, visual cues were offered with objects to assist in the example and teach strategies to the participants. As the individual performed the activity more easily, assistance was gradually reduced and eventually eliminated. There were six syllabic exclusion items and six phonemic exclusion items per session.

* Rapid naming to increase the velocity of lexical access [24]: Two lists from the PRONAR-LE remediation program with quick, automatic naming and reading [24] were presented in each session. The researcher first named and/or read the stimuli together with the participant, who was instructed to name or read each figure or linguistic item (objects, words, or pseudowords) as quickly and correctly as possible. When more than five errors were committed, the list was presented a second time with the instruction to read it again more carefully. If performed very slowly, the instruction was given to perform it again at a faster pace. Each list was performed a maximum of twice.

* Operational memory - digits and letters [25]: sequences of numbers and letters in random order were presented orally and the participant was asked to rearrange and repeat the sequences in alphabetical order and increasing numerical order. Visual assistance was given at the beginning and the number of stimuli was increased based on a $50 \%$ rate of correctly performing of the task.

* Reading words on a computer program with the aim of increasing reading velocity and word precision using the phonological and lexical routes [26]: Words were presented in segmented form (in letters) and complete form three consecutive times. The presentation time was $500 \mathrm{~ms}$ per stimulus (letter or word) in the first five sessions, as suggested by Nepomucemo \& Avila (2013) [26] and was reduced to $350 \mathrm{~ms}$ in the second week. The words were taken from a reading database for primary schoolchildren [27] and separated into high, medium, and low frequency. The words were presented randomly, with 40 different words presented in each session.

* Reading from a list of words (duration: three to five minutes): At the end of each session (without the tDCS electrodes), the participants read aloud the same list of words used in the program with the aim of increasing reading rate and accuracy through increasing the automatic recognition of words.

Five 30-minute sessions of combined stimulation were held, followed by a two-day interval and another five consecutive sessions. None of the participants took part in any other intervention program during the six months after intervention unless oriented to do so.

Friedman's test was used to compare variables among the different evaluation times (significance $<0.05$ ). The JT method [28] was used to determine the clinical significance (external validity) and measure the impact of the intervention program (whether a change in clinical status occurred from a dysfunctional to functional performance). The reliable change index (internal validity) was investigated to determine whether the changes were attributed to the procedures applied or merely fluctuations, artifacts, or measurement errors.

\section{Results}

The Table 1 displays individual performance values at each evaluation moment. Except for speed of reading isolated words, the 4 participants increased the speed and the number of correct answers in Assessment 6, compared to Assessment 0. As for text reading, all showed improvement since Assessment 1 and continued to improve until the Assessment 6, even without intervention.

When observing the group, despite the number of participants being small, there was an improvement in fluency, with a significant increase in both rate and accuracy $(\mathrm{p}=0.046)$ of text reading one month after the intervention. A significant increase in the accuracy of reading words and words plus pseudowords was found six months after the initial assessment $(\mathrm{p}=0.046)$. 
Table 1 Individual values in reading tasks in each assessment and comparison of performance averages in the evaluation after 01 months and after 06 months, in relation to the initial evaluation

\begin{tabular}{|c|c|c|c|c|c|c|c|c|c|c|c|c|c|c|}
\hline \multirow{3}{*}{ Tasks } & \multicolumn{12}{|c|}{ Participants } & \multirow{2}{*}{\multicolumn{2}{|c|}{$P$ value }} \\
\hline & \multicolumn{3}{|c|}{1} & \multicolumn{3}{|c|}{2} & \multicolumn{3}{|c|}{3} & \multicolumn{3}{|c|}{4} & & \\
\hline & Av0 & Av 1 & Av 6 & Av0 & Av 1 & Av 6 & Av0 & Av 1 & Av 6 & Av0 & Av 1 & Av 6 & Av 1 & Av 6 \\
\hline Accuracy w. & 29 & 33 & 38 & 34 & 38 & 36 & 21 & 25 & 28 & 30 & 30 & 38 & 0,083 & $0,046^{*}$ \\
\hline $\begin{array}{l}\text { Accuracy } \\
\text { pw. }\end{array}$ & 4 & 8 & 10 & 6 & 10 & 12 & 7 & 11 & 10 & 9 & 7 & 13 & 0,105 & 0,105 \\
\hline total & 33 & 41 & 48 & 40 & 48 & 48 & 28 & 36 & 38 & 39 & 37 & 51 & 0,317 & $0,046^{*}$ \\
\hline Word rate & 24 & 26,66 & 27,42 & 30,31 & 32,35 & 48,8 & 34,61 & 25,04 & 31,3 & 35,12 & 43,63 & 41,7 & 0,368 & 0,368 \\
\hline $\begin{array}{l}\text { Word } \\
\text { accuracy }\end{array}$ & 10 & 15,55 & 20,05 & 18,95 & 22,59 & 38,6 & 14,61 & 13,56 & 16,3 & 16,82 & 29,09 & 33,9 & 0,317 & $0,046^{*}$ \\
\hline Text rate & 60,2 & 66 & 76,5 & 76 & 78 & 79,01 & 62,89 & 83,82 & 67,3 & 79,22 & 92 & 99 & $0,046^{*}$ & $0,046^{*}$ \\
\hline $\begin{array}{l}\text { Text } \\
\text { accuracy }\end{array}$ & 48,9 & 57,7 & 69,6 & 56 & 65 & 68,5 & 44,21 & 70,58 & 59,1 & 60,66 & 88 & 96,8 & $0,046^{*}$ & $0,046^{*}$ \\
\hline \multicolumn{15}{|c|}{$\begin{array}{l}\text { Legend: Friedman Test; significance } \mathrm{p}<0.05 \\
\text { Abbreviations: } w=\text { words: } \mathrm{pw}=\text { pseudov }\end{array}$} \\
\hline
\end{tabular}

The reliable change and clinical significance indices for each participant and each variable are displayed in Table 2. Most changes (increase in reading rate and accuracy of words, pseudowords, and text) were attributed to the intervention program. Changes in status were found in the population from dysfunctional to functional for different variables (clinical significance).

Table 2 Reliable Change (RC) and Clinical Significance (CS) by participant, for the variables number of correct answers in word reading, pseudowords and rate and accuracy of words in the pre, one-month and six- month evaluation

\begin{tabular}{|c|c|c|c|c|c|c|c|c|c|}
\hline \multicolumn{2}{|l|}{ Participant } & \multicolumn{2}{|c|}{1} & \multicolumn{2}{|c|}{2} & \multicolumn{2}{|c|}{3} & \multicolumn{2}{|c|}{4} \\
\hline Gender & & \multicolumn{2}{|c|}{ Female } & \multicolumn{2}{|c|}{ Female } & \multicolumn{2}{|c|}{ Male } & \multicolumn{2}{|c|}{ Female } \\
\hline Age (years) & & \multicolumn{2}{|c|}{08.8} & \multicolumn{2}{|c|}{09.1} & \multicolumn{2}{|c|}{14.3} & \multicolumn{2}{|c|}{12.6} \\
\hline Revaluation & & 01 month & 06 months & 01 month & 06 months & 01 month & 06 months & 01 month & 06 months \\
\hline \multirow[t]{2}{*}{ Scores on reading words + pseudowords } & $\mathrm{RC}$ & + & + & + & + & + & + & - & + \\
\hline & CS & & & & & & & & \\
\hline \multirow[t]{2}{*}{ Scores on reading regular words } & $\mathrm{RC}$ & + & + & + & + & & + & & + \\
\hline & CS & & + & + & + & & + & & + \\
\hline \multirow[t]{2}{*}{ Scores on reading irregular words } & $\mathrm{RC}$ & - & + & + & + & + & + & & + \\
\hline & CS & & + & + & + & & & & + \\
\hline \multirow[t]{2}{*}{ Scores on reading pseudowords } & $\mathrm{RC}$ & + & + & + & + & + & + & - & + \\
\hline & CS & & & & & & & & \\
\hline \multirow[t]{2}{*}{ Word rate } & $\mathrm{RC}$ & + & + & + & + & & & + & + \\
\hline & $\mathrm{CS}$ & & & & + & & & & \\
\hline \multirow[t]{2}{*}{ Word Accuracy } & $\mathrm{RC}$ & + & + & + & + & + & + & - & \\
\hline & CS & & + & & + & & & & \\
\hline
\end{tabular}

At the six-month evaluation, all participants exhibited a clinically significant improvement in reading of common words, three exhibited improvement in uncommon words reading, and two changed statuses in terms of accuracy. One participant exhibited a clinically significant improvement in both common and uncommon words at the one-month evaluation and was the only one to change status in terms of word reading rate (after six months).

Regarding side effects, during stimulation sessions the participants described an itching sensation in the region stimulated and one participant reported migraine after the first two stimulation sessions. 


\section{Discussion}

Reading fluency (rate and accuracy) was the focus of the stimulation in the present study. Based on Friedman test results, improvements were found in text reading velocity one-month after tDCS, but no improvement was found in reading rate of isolated words. The participants were stimulated through two reading routes (phonological and orthographic), as the words were presented segmented and then whole. In one of the cases, reading time and accuracy increased, demonstrating an improvement in the phonological route, for which a deficit had previously been found. Moreover, clinically significant increase in accuracy score was found, especially for reading common words, demonstrating the use of orthographic route by the four participants.

Therefore, improvements in reading fluency were attributed to improvements in using two routes. However, these findings differ from data described in previous studies, which prioritized phonological route during the placement of anodal electrode (temporal-parietal region) tested either alone [15] or in combination with cognitive training involving emphasis on grapheme-phoneme correspondence [14]. In the present study, the parieto-occipital region (P5) was stimulated, which is related to magnocellular deficit [29] associated with areas responsible for phonological processing and grapheme-phoneme correspondence [30]. The decision was made not to inhibit contralateral areas, as recent studies have shown that children at risk and those with DD have greater activation of the contralateral areas, compensating for deficits in left brain [3,31].

We should also consider the fact that tDCS is not a focal form of stimulation [32,33] and the current may therefore have dissipated, leading to stimulation of other areas underlying the parieto-occipital region, such as temporal-parietal and temporo-parieto-occipital junctions. According to neuroimaging studies, these areas are under-activated in children with DD and are also important to phonological awareness tasks and, consequently, reading [2,3,31].

Besides tDCS, one must consider the type of cognitive-linguistic stimulation applied. Few studies have adopted this combination $[14,33]$. In the present investigation, a generic, multilevel reading stimulation therapy was designed with different skills related to reading learning that are recognized as being deficient in children with DD. Moreover, three theories to explain the neurological basis of DD were considered. The phonological deficit therapy postulates that DD is associated with changes and hypoactivation of circuits in the temporal, temporo-occipital, and temporo-parietal cortex. The second theory is that dyslexia originates from changes in the magnocellular system, which is involved in visual attention, eye movements, and spatial orientation and is associated with the posterior parietal cortex. The third theory is about cerebellar deficit and highlights the participation of the cerebellum in balance as well as the language processing and automatisms [28]. This could explain the greater improvements in different variables found in comparison to previous studies $[13,14]$.

The individual analyses using the JT method confirmed the effectiveness of the combined interventions, as most changes were reliable, and all participants achieved the minimum values of normality regarding accuracy for common words and some uncommon words as well as fluency in the reading of isolated words.

The participants exhibited a good overall performance in both short and long term, demonstrating the effects of metaplasticity [13,34], as the significant improvement in reading was maintained after six months. This underscores the relevance of the present findings, as the literature shows that interventions for DD tend to be long term [35]. However, although positive and promising, the results cannot be generalized, as the sample was small and heterogeneous in terms of age and school grade.

The present study has limitations that should be considered. The sample was small, and the heterogeneity of the participants does not enable the generalization of the results. Another limitation was the lack of control groups to determine the effects of active tDCS, sham tDCS, active tDCS alone (not combined with cognitive therapy), and cognitive therapy alone (without tDCS), as the reading therapy program was not tested alone to determine its effects and most of the participants in the study were not undergoing language therapy. The consensus in the literature is that treatment for DD tends to be long [35]. The placement of the cathodal electrode should also be studied to determine the best stimulation site (areas of the contralateral hemisphere, the arm, or buccinator muscle). Another limitation of this study was the failure to determine the subtypes of dyslexia among the participants, which could explain some of the results and could have assisted in the choice of electrode placement and the direction of therapy.

Controlled, blinded studies could assist in determining the best areas of stimulation and inhibition to determine the contribution and weight of each type of stimulation (tDCS and reading stimulation program). 


\section{Conclusion}

All the participants showed positive short- and long-term effects on reading fluency and accuracy after received the combination of anodal tDCS administered over parietal-occipital area with cathode placed over contralateral deltoid muscle combined with a multilevel reading stimulation program (stimulation of reading and predictive skills). This combination proved to be promising for a rehabilitation that has DD. However, the small sample size prevents generalization of the results.

\section{Compliance with ethical standards}

\section{Acknowledgments}

We would like to thank the following people for their contribution to this research: the graduate students of Núcleo de Assistência, Ensino e Pesquisa em Escrita e Leitura (NEAPEL), Núcleo de Atendimento Neuropsicológico Infantil Interdisciplinar (NANI) and Laboratório de Neuromodulação e Desenvolvimento (LAND): Suélen Graton, Gabriela Bueno, Mariana Oliveira, Esther Simonato, Denise Vettorazzo, Luzinete Sá, Mariana Castro and to Nailê Oliveira (speechlanguage therapist) and Patricia Oliveira - PhD (psychologist).

\section{Disclosure of conflict of interest}

The Authors declares that there is no conflict of interest.

\section{Funding}

This research was funded by the Coordination of Superior Level Staff Improvement (CAPES), Ordinance no 206.

\section{References}

[1] American Psychiatric Association et. al. DSM5: Diagnostic and statistical manual of mental disorders. Artmed Editora, 2014; (5): 31-86.

[2] Buchweitz A, Costa AC, Toazza R, Moraes AB, Cara VM, Esper NB, Aguzzoli C, Gregolim B, Dresch LF, Soldatelli MD, Costa JC, Portuguez MW, Franco AR. Decoupling of the Occipitotemporal Cortex and the Brain's Default-Mode Network in Dyslexia and a Role for the Cingulate Cortex in Good Readers: A Brain Imaging Study of Brazilian Children. Developmental neuropsychology. 2019; 44(1): 146-157.

[3] Luniewska M, Chyl K, Dębska A, Banaszkiewicz A, Żelechowska A, Marchewka A, Grabowska A, Jednoróg K. Children With Dyslexia and Familial Risk for Dyslexia Present Atypical Development of the Neuronal Phonological Network. Front. Neurosci. 2019; 13: 1287.

[4] Ziegler JC, Castel C, Pech-Georgel C, George F, Alario FX, Perry C. Developmental dyslexia and the dual route model of reading: simulating individual differences and subtypes. Cognition. 2008; 107(1): 151-178.

[5] Friedmann N, Coltheart M. Types of developmental dyslexia. In A. Bar-On, \& D. Ravid (Eds.), Handbook of communication disorders: Theoretical, empirical, and applied linguistic perspectives Berlin, Boston: De Gruyter Mouton. ISBN: 978-1-61451-685-9. ton: De Gruyter Mouton. 2018; 721-751.

[6] Nogueira DM, Carnio MS. Speech therapy program in reading comprehension and spelling: effects on spelling in dyslexics. CoDAS. 2018; 30(2): e20170077.

[7] Cidrim L, Madeiro F. Spelling studies in dyslexia: literature review. J. CEFAC. 2017 Nov-Dec; $19(6): 842-854$.

[8] Richlan F. Functional neuroanatomy of developmental dyslexia: The role of orthographic depth. Frontiers in Human Neuroscience. 2019; 8: 347.

[9] Wagner, RK, Torgesen, JK. The nature of phonological processing and its causal role in the acquisition of reading skills. Psychological Bulletin. 1987; 101(2): 192-212.

[10] César ABPC, Capellini SA. Multisensory intervention speech therapy program for subjects with dyslexia: application and clinical significance. Marilia. [Dissertation]. UNESP. 2018.

[11] Horowitz-Kraus T. Pinpointing the deficit in executive functions in adolescents with dyslexia performing the Wisconsin Card Sorting Test An ERP Study. Journal of Learning Disabilities, Riverside, USA. 2014; 47(3): 208223. 
[12] D'Mello AM, Turkeltaub PE, Stoodley CJ. Cerebellar tdcs modulates neural circuits during semantic prediction: a combined tDCS-fMRI study. J. Neurosci. 2017; 37: 1604-1613.

[13] Fregni F, Bossio PS, Brunoni AR. Therapeutic neuromodulation: principles and advances in noninvasive stimulation in neurology, rehabilitation, psychiatry and neuropsychology. Sarvier. São Paulo. 2012.

[14] Cancer A, Antonietti A. tDCS modulatory effect on reading processes: a review of studies on typical readers and individuals with dyslexia. Front. Behav. Neurosci. 2018; 12: 162.

[15] Rios DM, Rios MC, Bandeira ID, Campbell FQ, Vaz DC, Lucena R. Impact of Transcranial Direct Current Stimulation on Reading Skills of Children and Adolescents With Dyslexia. Child Neurology Open. 2018; 5: 1-8.

[16] Costanzo F, Rossi S, Varuzza C, Varvara P, Vicari S, Menghini D. Long-lasting improvement following tDCS treatment combined with a training for reading in children and adolescents with dyslexia. J. Neuropsychologia. 2018; 130: 38-43.

[17] Lima AL, Braga FMA, Costa RMM, Gomes EP, Brunoni AR, Pegado R. Transcranial direct current stimulation for the treatment of generalized anxiety disorder: A randomized clinical trial. Journal of Affective Disorders. 2019; 259: 31-37.

[18] Lúcio PS, Cogo-Moreira H, Kida ASB, Carvalho CAF, Pinheiro AMV, Mari JJ, Ávila CRB. Word decoding task: Item analysis byIRT and within-group norms. Psicologia: Teoria e Pesquisa. 2018; 34: e3437.

[19] Carvalho CAF, Avila CRB. Pragmatic function of language and reading comprehension in dyslexia. São Paulo. [dissertação]. UNIFESP. 2013.

[20] Salles JF, Piccolo RL, Miná CS. Anele Collection 1 - Isolated Word and Pseudoword Reading Assessment- LPI. 2017.

[21] Moura 0, Simoes MR, Pereira M. Semantic and phonemic verbal fluency in children: cognitive functions and temporal analysis. Ev. psychol., Itatiba , 2013; 12 (2): 167-177.

[22] Bugalho P, Correa B, Viana-Baptista M. Role of the cerebellum in cognitive and behavioral functions: scientific bases and study models. Acta Médica Portuguesa. 2006; 19: 257-268.

[23] Capellini SA, Sampaio MN, Kawasaki KHS; Padula, NAMR, Santos LCA, Lorencetti MD, Smythe I. Therapeutic efficacy of the phonological remediation program in students with developmental dyslexia. RevCefac, 2009.

[24] Santos B, Capellini SA. Intervention program with rapid automatic naming and reading: pilot study. Marilia. [Dissertation] UNESP. 2017.

[25] Medina GBK, Guimaraes SR. Reading by Students with Developmental Dyslexia: Impacts of an Intervention with Phonic Method Associated with Stimulation of Executive Functions. J. bras. educ. espec. 2019. 25(1): $155-174$.

[26] Nepomuceno PF, Ávila CRB. Characterization of the performance of students with and without reading difficulties in reading decoding tasks. CoDAS. 2013; 25(4): 330-6.

[27] Oliveira AM, Capellini SA. E-READING II: word bank for reading by Elementary School II students. CoDAS. 2016; 28(6): 778-817.

[28] Jacobson NS, Truax P. Clinical significance: a statistical approach to defining meaningful change in psychotherapy research. J Consult Clin Psychol. [Internet]. 1991; 59(1): 12-9.

[29] Jaskowski P, Rusiak P. Posterior parietal cortex and developmental dyslexia. CTA NEUROBIOL EXP. Polônia. 2015; 65: 79-94.

[30] Pugh KR, Mencl WE, Jenner AR, Katz L, Frost SJ, Lee JR, et al. Functional neuroimaging studies of reading and reading disability(developmental dyslexia). Ment. Retard. Dev. Disabil. Res. Rev. 2000; 6: 207-213.

[31] D`Mello AM, Gabrieli JDE. Cognitiva Neuroscience of Dyslexia. Linguagem, Speech, and Hearing Services in Schools. 2018; 49: 796-809.

[32] Woods AJ, Antal A, Bikson M, Boggio PS, Brunoni AR, Celnik P, Cohen LG, Fregni F, Herrmann CS, Kappenman ES, Knotkova H, Liebetanz D, Miniussi C, Miranda PC, Paulus W, Priori A, Reato D, Stagg C, Wenderoth N, Nitsche MA. A technical guide to tDCS, and related non-invasive brain stimulation tools. Clinical neurophysiology: official journal of the International Federation of Clinical Neurophysiology. 2016; 127(2): 1031-1048.

[33] Zhang Y, Song H, Chen Y, Zuo L, Xia X, Zhang X. Thinking on Transcranial Direct Current Stimulation (tDCS) in Reading Interventions: Recommendations for Future Research Directions. Front. Hum. Neurosci. 2019; $13: 157$. 
[34] Kronberg G, Bridi M, Abel T, Bikson M, Parra LC. Direct Current Stimulation Modulates LTP and LTD: Activity Dependence and Dendritic Effects. Brain stimulation. 2017; 10(1): 51-58.

[35] Horowitz-Kraus T, Finucane S. Separating the Different Domains of Reading Intervention Programs: A Review. SAGE Open. 2016; 6(2). 